\title{
Back to the Future: Preserved Hippocampal Network Activity during Reverse Ambulation
}

\author{
Andrew P. Maurer, ${ }^{1,2,4}$ Adam W. Lester, ${ }^{1,2}$ Sara N. Burke, ${ }^{1,2,4}$ Jonathan J. Ferng, ${ }^{1,2}$ and Carol A. Barnes ${ }^{1,2,3}$ \\ ${ }^{1}$ Evelyn F. McKnight Brain Institute, ${ }^{2}$ Arizona Research Laboratories Division of Neural Systems, Memory and Aging, and ${ }^{3}$ Departments of Psychology, \\ Neurology, and Neuroscience University of Arizona, Tucson, Arizona 85724, and ${ }^{4}$ Department of Neuroscience, College of Medicine, University of Florida, \\ Gainesville, Florida 32611
}

During movement, there is a transition of activity across the population, such that place-field centers ahead of the rat are sequentially activated in the order that they will be encountered. Although the mechanisms responsible for this sequence updating are unknown, two classes of models can be considered. The first class involves head-direction information for activating neurons in the order that their place fields will be traversed. An alternative model contends that motion and turn-related information from the posterior parietal cortex shift the subset of active hippocampal cells across the population. To explicitly test these two models, rodents were trained to run backward on a linear track, placing movement in opposition with head orientation. Although head-direction did not change between running conditions, place-field activity remapped and there was an increase in place-field size during backward running compared with forward. The population activity, however, could still be used to reconstruct the location of the rat accurately. Moreover, theta phase precession was maintained in both running conditions, indicating preservation of place-field sequences on short-time scales. The observation that sequence encoding persists even when the animal is orientated away from the direction of movement favors the concept that posterior parietal cortical mechanisms may be partially responsible for updating hippocampal activity patterns.

Key words: oscillations; phase precession; place cells; population vector; sequence learning; theta

\section{Introduction}

During movement through an environment, principal cells of the hippocampus express a firing pattern tuned to spatial location, referred to as the neuron's place field (O'Keefe and Dostrovsky, 1971). This is accompanied by a prominent $4-12 \mathrm{~Hz}$ oscillation in the local field potential, termed the theta rhythm (Vanderwolf, 1969; Buzsàki and Eidelberg, 1983). Importantly, within each theta cycle, the active population of neurons with common place fields dynamically changes (O'Keefe and Recce, 1993; Harris, 2005). This propagation of activity is sequentially organized, such that neurons with maximum firing behind the rat fire before neurons with place fields ahead of the rat, providing a "lookahead" of upcoming spatial locations (Burgess et al., 1994; Skaggs et al., 1996; Tsodyks et al., 1996; Maurer et al., 2012). Furthermore, between adjacent theta cycles, as neurons with receptive field locations behind the rat become inactive, cells with fields representing locations ahead of the animal begin to fire. The consequence of the sequential updating of active cell assemblies

Received March 19, 2014; revised Sept. 16, 2014; accepted Sept. 21, 2014.

Author contributions: A.P.M. and C.A.B. designed research; A.W.L., S.N.B., and J.J.F. performed research; A.P.M. and A.W.L. analyzed data; A.P.M., A.W.L., S.N.B., and C.A.B. wrote the paper.

This work was supported by the McKnight Brain Research Foundation, and NIH Grants AG012609 and NS070464. We thank Kim Bohne, Lan Hoang, Jie Wang, Michael Montgomery, Michelle Carroll, and Luann Snyder for help with completing this paper.

The authors declare no competing financial interests.

Correspondence should be addressed to Carol A. Barnes, University of Arizona, Evelyn F. McKnight Brain Institute, Life Sciences North Building, Room 355, Tucson, AZ 85724-5115. E-mail: caro@@nsma.arizona.edu.

DOI:10.1523/JNEUROSCI.1129-14.2014

Copyright $\odot 2014$ the authors $\quad 0270-6474 / 14 / 3415022-10 \$ 15.00 / 0$ are that neurons will exhibit a progressive shifting of spikes relative to the hippocampal theta rhythm (Dragoi and Buzsàki, 2006; Foster and Wilson, 2007; Geisler et al., 2010; Maurer et al., 2012). This phenomenon, termed theta phase precession (O'Keefe and Recce, 1993), is thought to be a fundamental mechanism of spatial ensemble sequence encoding (Lisman and Idiart, 1995; Jensen and Lisman, 1996; Skaggs et al., 1996; Lisman and Buzsàki, 2008), as well as nonspatial information (Harris et al., 2002; Harris, 2005), such as episodic recall and action planning (Pastalkova et al., 2008). The afferent drive responsible for updating hippocampal representations, and thus phase precession, however, are unknown.

During a random foraging task, it has been shown that the look-ahead is selectively oriented toward the rodent's upcoming movement direction, suggesting that head-direction is a primary mechanism for updating network activity (Huxter et al., 2008). Moreover, attractor models of hippocampal activity dynamics traditionally use a combination of head-direction (Taube et al., 1990) and self-motion information to move the distribution of activity in the network (Redish and Touretzky, 1997; Samsonovich and McNaughton, 1997; McNaughton et al., 2006). These models place a significant dependence on the egocentric headdirection signal, necessitating that the movement trajectory is aligned with head orientation to appropriately move the "bump" of activity. Alternatively, velocity and motion information from the parietal cortex could be responsible for appropriately updating the hippocampal network (Conklin and Eliasmith, 2005). As the activity of neurons in the posterior parietal cortex are sensi- 
tive to simple motion states (McNaughton et al., 1994; Burke et al., 2005) that precede the actual onset of movement up to $500 \mathrm{~ms}$ (Whitlock et al., 2012). Thus, anticipatory motor planning could be responsible for the hippocampal "look ahead" phenomenon (Maurer and McNaughton, 2007). By training rats to run forward, as well as backward, on a linear track, the present study sought to test the dissociating predictions of the two models when a rodent's movement trajectory is decoupled from the direction that it was currently facing (Conklin and Eliasmith, 2005).

\section{Materials and Methods}

Animals and surgical procedures. Four Fischer-344 male rats (8- to 12months-old, RRID: F344/DuCrl Rat) were used in this study. Rats were housed individually and maintained on a $12 \mathrm{~h}$ light/dark cycle. Recordings took place during the dark phase. Surgery was conducted according to NIH guidelines for rodents and approved Institutional Animal Care and Use Committee protocols. The rats were implanted, under isoflurane anesthesia, with a "hyperdrive" array of 12 separately moveable tetrodes. Two additional tetrodes, with wires shorted together, served as references. For all rats, hippocampal recordings were made from CA1 ( $3.0 \mathrm{~mm}$ posterior to bregma, $1.4 \mathrm{~mm}$ lateral to the midline).

Electrophysiological recording. After surgery, tetrodes were lowered and allowed to stabilize for several days above CA1, and then gradually advanced into the stratum pyramidale. A reference electrode was placed in or near the corpus callosum. Each tetrode was attached to four separate channels of a 50-channel unity-gain head stage (Neuralynx). A multiwire cable connected the head stage to digitally programmable amplifiers (Neuralynx). The spike signals were amplified (gain of 1000-5000), bandpass filtered between $600 \mathrm{~Hz}$ and $6 \mathrm{kHz}$, and transmitted to the Cheetah Data Acquisition system (Neuralynx). Signals were digitized at $32 \mathrm{kHz}$, and events that reached a predetermined threshold were recorded for $1 \mathrm{~ms}$. Spikes were sorted offline by means of the semiautomatic clustering algorithm KlustaKwik (K. D. Harris, University College, London, RRID: nif-0000-10182). The resulting classification was refined manually with custom-written software (MClust; A. D. Redish, University of Minnesota), resulting in spike-train time series for each of the well isolated cells.

Pyramidal neurons were identified using the standard parameters of firing rate, burstiness, spike waveform characteristics (Ranck, 1973), and the first moment of the autocorrelation (Csicsvari et al., 1998). EEG signals were bandpass filtered between 1 and $300 \mathrm{~Hz}$, sampled at $2.4 \mathrm{kHz}$, and amplified on the head stage with unity gain, and then again with variable gain amplifiers (up to 5000). Several light-emitting diodes were mounted on the head stage to allow position tracking. The position of the diode array was detected by a television camera placed above the experimental apparatus, recorded with a sampling frequency of $60 \mathrm{~Hz}$, and a spatial resolution was $0.2 \mathrm{~cm} /$ pixel.

Behavior. The animals were food deprived to $85 \%$ of ad libitum weight. During this time, rats were shaped to walk forward and backward on a narrow linear track. The rat was placed on the track and allowed to run forward to obtain food reward. After eating, without letting the rats turn around, the trainer would gently place a plastic spoon against their nose. If the rat stepped back, a small food-reward was immediately presented. This shaping continued until the rats were fully trained to cover the entire track for reward. Recording began after surgery recovery. Each running session was bookended by a rest epoch of at least $20 \mathrm{~min}$ to assess baseline firing and stability.

Analyses. Instantaneous velocity was determined by convolving the position data over multiple frames and then calculating the absolute value of the difference in position. Track locations where occupancy exceeded $10 \%$ of the total time were removed, which was always associated with reward. The position data were then "unwrapped" to examine forward and reverse directions separately. Lap duration was calculated as the time from leaving one reward location and entering the other rewarded area.

Place-field diagrams and phase-precession diagrams (Fig. 1) were constructed as described by Maurer et al. (2006a,b). Briefly, each running
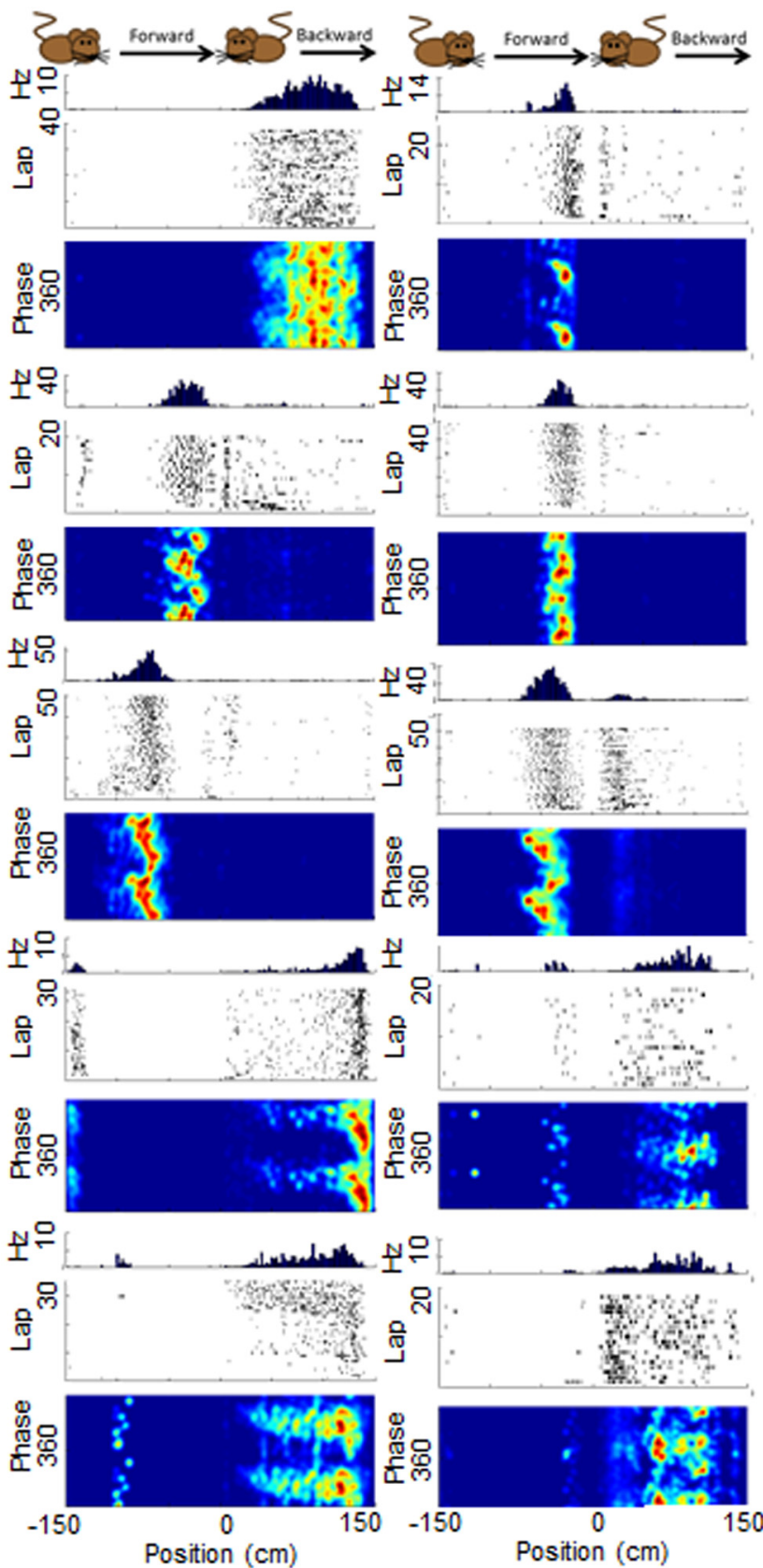

Figure 1. Representative place-field activity during different running behaviors. Examples of 10 pyramidal neurons during forward (running from -150 to $0 \mathrm{~cm}$ ) and backward $(0$ to $+150 \mathrm{~cm})$ conditions. For example, -100 and $+100 \mathrm{~cm}$ correspond to the same location on the track, but reflect when the rat is locomoting forward $(-)$ versus backward $(+)$, respectively. The top panels show occupancy normalized firing rate by position. The middle shows spike rasters by position, and the bottom shows traditional occupancynormalized spike-phase density plots showing theta phase by location on the track (theta phase is plotted twice to account for circularity). Note that areas on the track with higher firing rates are not symmetrically aligned around the 0 location, which corresponds to the point in which the movement trajectory changed from forward to backward running ( $\pm 150 \mathrm{~cm}$ is the location in which running changes from backward to forward). This qualitatively illustrates the lack of bidirectional activity between different running conditions. Furthermore, these plots show the tendency for place-field spiking during backward running to shift location across subsequent laps, despite the larger field size (right column, bottom two examples). The inconsistency of firing during backward locomotion renders the use of traditional phase-location measures of precession inappropriate. 
trajectory, forwards versus backward ambulation were plotted separately to control for the unidirectional nature of place fields during track running (McNaughton et al., 1983). Spikes were binned by location on the track into $2.5 \mathrm{~cm}$ bin widths, and rates were normalized by bin occupancy time (Maurer et al., 2006a,b). To construct the theta phase of firing versus position plots, each spike was assigned a phase relative to the hippocampal theta rhythm using methods similar to those of Belluscio et al. (2012; described below). The spikes were binned into a two dimensional matrix, convolved, and normalized by occupancy to provide an occupancy normalized phase-position density plot. To display the position of each spike, lap-by-lap spike rasters were created by linearly interpolating the spike times of a neuron onto the rodents position-and-time matrix.

Spatial population vectors were constructed in the same manner as by Maurer et al. (2012) with the exception that spatial bins were reduced to $0.33 \mathrm{~cm}$ (compared with $0.7 \mathrm{~cm}$ ) and were generated on a lap-by-lap basis. The rationale for constructing lap-by-lap population vectors, as opposed to collapsing across all laps, was to attempt to control for the higher variability in the mean position of spikes across laps in backwards running. Briefly, to generate the population vector matrix, the spatial firing rate distributions of all recorded pyramidal neurons in a given condition were combined into a single, two-dimensional array: cell number in rows and linearized location in the columns. Each column therefore represented an estimate of the composite population vector for the corresponding location. The vectors for the forward and reverse running directions were computed separately. To reconstruct the position of the rat, one spatial population vector matrix was selected as the reference and was then correlated against every other spatial population vector for the other laps. This process was repeated until each lap was correlated against all other laps, excluding the autocorrelation of the reference lap with itself, providing a reconstructed position for each lap-combination.

Inspection of our data using the lap-by-lap rastergrams and phase by position density plots (Fig. 1), suggests that during backward running, place fields may undergo a mild drift or dispersion. This observation is similar to findings from experiments in which rodents forage in dark arenas (Markus et al., 1994; Hafting et al., 2005) or when landmarks are perceived to be unstable (Knierim et al., 1995). To measure the consistency of place-field location on a lap-to-lap basis, we applied firing rate boundaries to the spatial firing rate distributions (Muller et al., 1987; Thompson and Best, 1990; cf. Maurer et al., 2006b). Spikes across all laps were binned ( $2.5 \mathrm{~cm}$ bin size) and occupancy normalized. To be included in the analysis, the firing rate of the neuron needed to exceed $10 \%$ of the mean firing rate for 11 or more contiguous bins (for a minimum placefield size of $27.5 \mathrm{~cm}$, which was below the size of place fields observed in forward running conditions estimated from the population vector correlation). Using the boundaries of the regions that passed the firing rate criteria, the lap-by-lap center of mass of firing in the field was calculated (Mehta et al., 1997; Shen et al., 1997; Ekstrom et al., 2001; Lee et al., 2004; Burke et al., 2008). To measure whether place-field centers varied from lap-to-lap, we calculated the SD of the center-of-mass across all laps. In the backward condition, the SD of the center-of-mass was significantly larger $(3.34 \mathrm{~cm}, \mathrm{SEM} \pm 0.16)$ relative to the forward running condition $\left(2.69 \mathrm{~cm}, \mathrm{SEM} \pm 0.17 ; t_{(3)}=3.05, p<0.05\right)$. This quantitatively supports our qualitative impression that the place field-location undergoes a modest jitter across laps in the backward running condition relative to forward running. In light of this, we considered methods of determining theta phase precession.

Although there were specific examples where spike phase versus position shows clear precession during backward running (Fig. 1, left column row 4) in most cases the plots of spike phase versus position during backward running did not show clear precession, due to the higher variability in place-field position during backward running. However, we demonstrate consistent theta phase precession versus time (see Figs. 3, 6).

Although not an explicit measure of theta phase precession, prior studies have used phase-position correlations to infer theta phase precession (Maurer et al., 2006b; Burke et al., 2008, 2011). This analysis, however, does not demonstrate that the spike times shift their phase relative to the hippocampal theta rhythm, but only provides a means to determine the slope of precession in space and the coefficient of determination (how well the variation in one variable explains the variance in another).

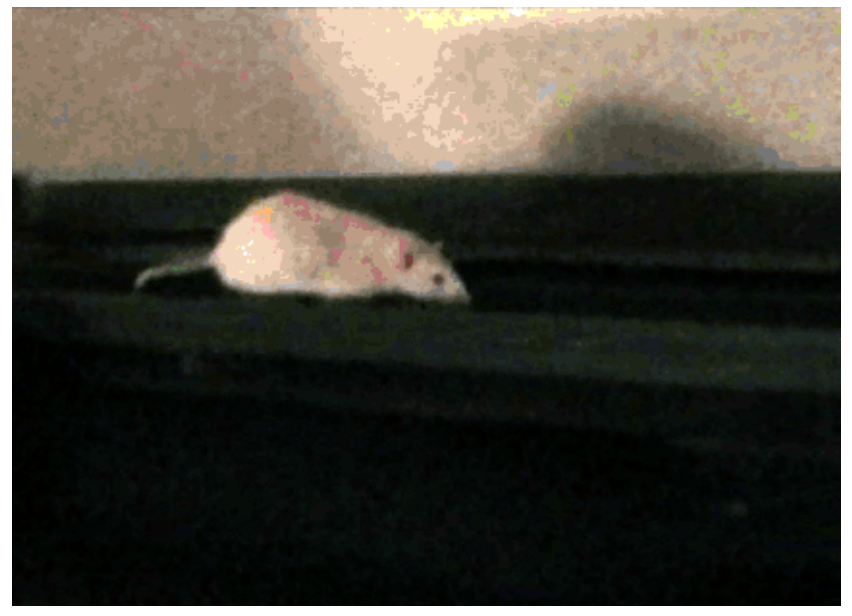

Movie 1. Video of a rat trained to run forward and backward, with the same head orientation, to obtain a food reward.

Moreover, as the center-of-mass is higher during backward running compared with forward traversals, meaning that the position of spikes cannot be treated as a fixed value (violating the weak exogeneity assumption). There are a number of other factors that make regression analysis an inappropriate model for determining spike phase-location relationships. These include the fact that linear-linear and circular-linear regression analyses are vulnerable to outliers in the data (Diba and Buzsàki, 2008), have difficulty in quantifying bimodal or nonlinear data (Kempter et al., 2012), and violate the basic statistical assumptions of homoscedasticity and normality. That is, phase variance is not equal across all positions (i.e., are not homoscedastic; Yamaguchi et al., 2002), and place fields can have a strong kurtosis (i.e., are not normally distributed; Mehta et al., 1997, 2000). Because of these factors, other techniques were required to quantify theta phase precession that are independent of position information (Harris et al., 2002; Pastalkova et al., 2008).

Our first nonspatial measure of theta phase precession was based on the analysis first developed by Harris et al. (2002), aimed at determining phase shifts that occur between the onset and offset of firing periods of neuronal spiking. For the present study, spikes in the backward and forward directions that belonged to an epoch when firing rate was increasing (the previous 8 theta cycles contained no more than 4 spikes, whereas the following 8 theta cycles contain at least 16 spikes) or decreasing (the previous 8 theta cycles contained 16 or more spikes, whereas the following 8 theta cycles contain 4 spikes or fewer; Harris et al., 2002) were determined. The rationale for these values is that, assuming theta is $8 \mathrm{~Hz}$, the spike frequency increases either from 4 to $16 \mathrm{~Hz}$ (or vice versa for the decreasing epochs). By calculating the mean circular phase of the onset and offset of spikes, it was possible to identify whether the spikes advanced relative to the theta rhythm.

The second phase precession analysis involved comparing the autocorrelogram frequency of individual neurons to the theta frequency across different running velocities (O’Keefe and Recce, 1993). To calculate the autocorrelograms by velocity, the animal's velocity at the occurrence of each spike was determined in bins of $10 \mathrm{~cm} / \mathrm{s}$. A moving window method was used to optimize the calculation of the spike autocorrelograms and theta peak autocorrelation frequency. To calculate the frequency of autocorrelograms, spectra were calculated using a multitaper method (Geisler et al., 2007).

Finally, a novel method for quantifying theta phase precession across the entire population of recorded CA1 cells was developed, the thetatime population vector autocorrelogram. This temporal population vector was constructed in a manner similar to the position population vector, with $n$ cells by $t$ temporal bins. Time was binned into $\sim 14^{\circ}$ of a theta cycle with 546 bins over 21 consecutive theta cycles centered on a single theta trough. The population vector within each theta bin was then correlated with all other bins across the 20 theta cycles. To account for the asymmetry of hippocampal theta oscillation (Buzsàki et al., 1983) in the 
A

\section{Spatial Population Vector Correlation:}

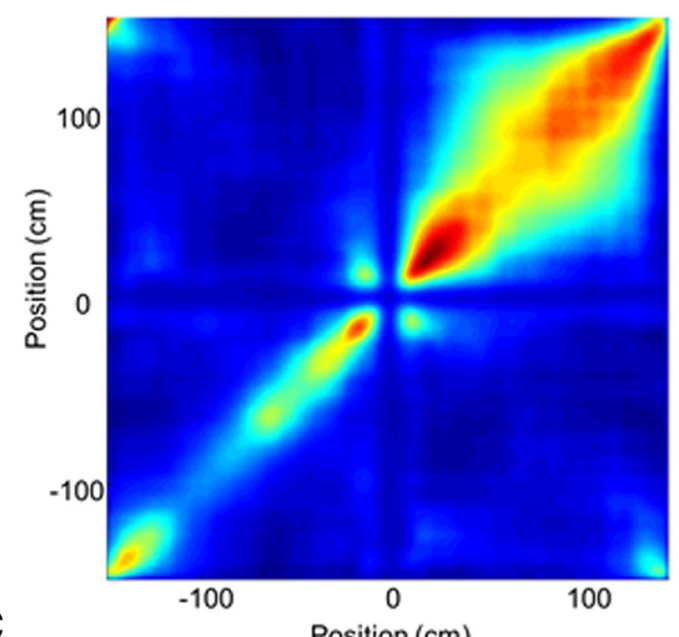

C

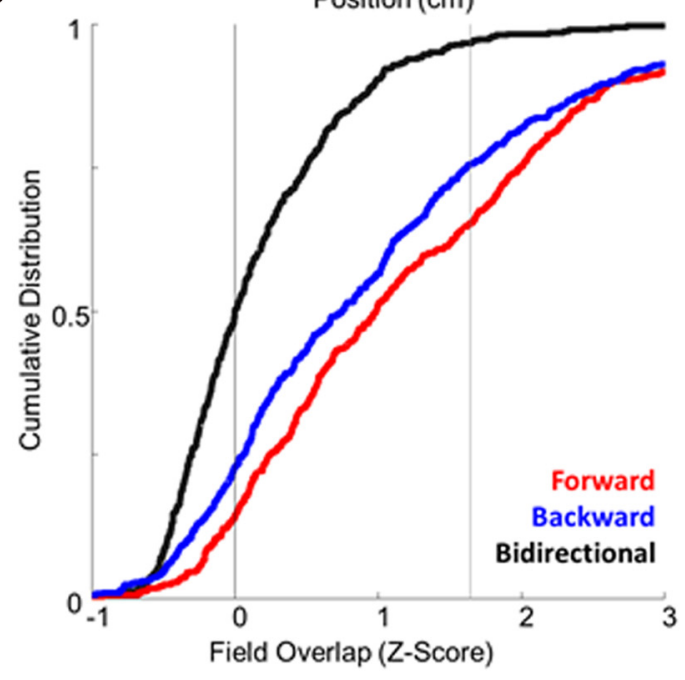

B

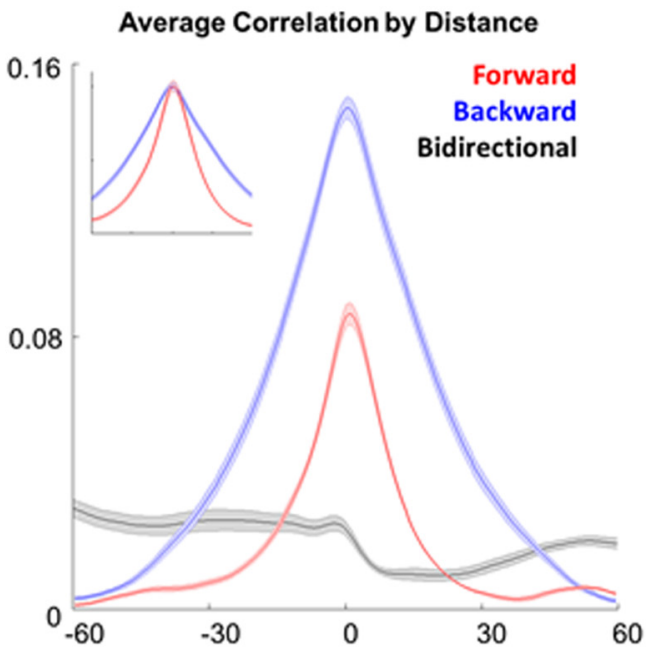

D

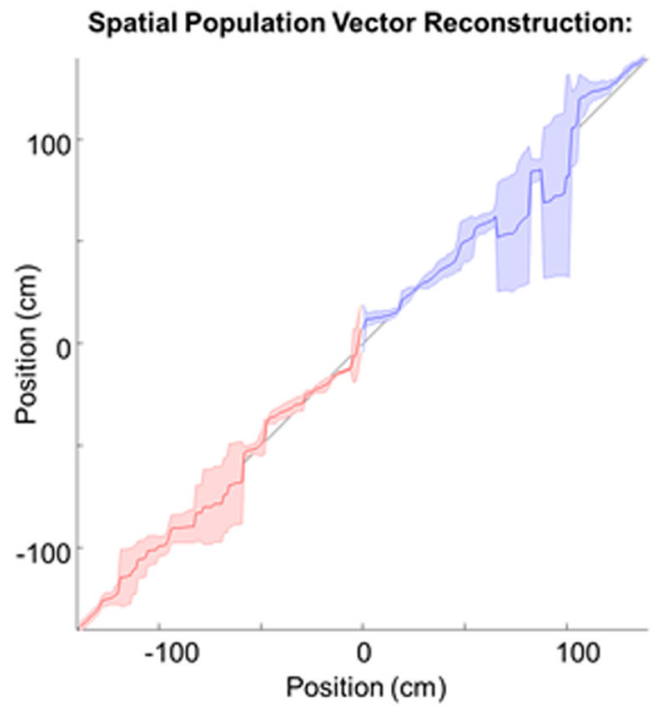

Figure 2. Spatial population vector correlation during forward and backward running. $A$, The mean population vector (PV) correlation matrices during forward (negative) and backward (positive) traversals for four rats. Warmer colors correspond to higher correlation values. $\boldsymbol{B}$, The mean PV correlation across laps for forward (red) and backward (blue) traversals. $\boldsymbol{B}$, Inset, The normalized PV correlations across laps. Average bidirectional (black) correlation values were significantly reduced relative to the correlation values across laps for the forward $(p<0.02)$ and backward $(p<0.001)$ running conditions alone. C, Cumulative Z-score distribution of place-field overlap between forward and backward runs (black; bidirectionality), forward-forward runs (red), and backwardbackward (blue) runs (place-field stability) relative to a shuffled control distribution. The vertical line at $Z=1.6$ corresponds to the proportion of fields with overlap above chance. The overall mean correlation across laps between the forward (mean: $0.19, \mathrm{SEM} \pm 0.02$ ) and backward (mean: $0.19, \mathrm{SEM} \pm 0.01$ ) directions was not significantly different from each other $(p=0.71)$. $\boldsymbol{D}$, The mean $\mathrm{PV}$ reconstructed position of rats plotted against actual position for both running conditions.

determination of neuronal firing phase, we used methods similar to those of Belluscio et al. (2012). The tetrode that recorded the most pyramidal neurons was selected as a theta reference. To "seed" our initial peak/ trough finding algorithm, we digitally filtered the EEG, bidirectionally in time to avoid phase shifts, with a $6-8 \mathrm{~Hz}$ Chebyshev bandpass filter. In these conditions, theta approximates a sinusoid facilitating peak determination by using the derivative of the oscillation. Next, we found the absolute minimum in the $1-80 \mathrm{~Hz}$ filtered EEG that fell between the time stamps of two peaks in the $6-8 \mathrm{~Hz}$ filtered data. These troughs were saved and used to find the absolute maximum in the $1-80 \mathrm{~Hz}$ trace that fell between the timestamps of two troughs (Belluscio et al., 2012).

Unless otherwise indicated, for all summary statistics means were calculated for each rat for comparisons between forward and backward running conditions and significance was tested using paired-samples $t$ tests with $\alpha \leq 0.05$. The positions at which rats receive reward were excluded from the analysis.

The degree to which the place fields showed stable location-specific firing on repeated runs in the same direction, as well as runs in opposite movement directions (bidirectionality) were assessed by calculating a standardized measurement of place-field overlap ( $S$; Battaglia et al., 2004) as follows:

$$
\begin{gathered}
S=\frac{2 \sum \min \left(\bar{P}_{\text {lap } i}, \bar{P}_{\text {lap } i+1}\right)}{\sum\left(\bar{P}_{\text {lap } i}, \bar{P}_{\text {lap } i+1}\right)}, \\
\bar{P}_{\text {lap }}=\frac{N_{\text {bins }} P_{\text {lap }}}{\sum\left(P_{\text {lap }}\right)} .
\end{gathered}
$$

$\bar{P}_{\text {lap }}$ is the normalized firing rate vector composed of the firing rate at each position bin on the linear track divided by the sum of firing rates across bins for a given lap. $\bar{P}_{\text {lap }}$ is calculated for all laps. This overlap measure is invariant for rescaling of the place-field profiles, and it assumes the limit values of 0 for nonoverlapping place-field profiles and of 1 for profiles that are identical. The s value was computed for all pairwise lap combinations. These values were then averaged for each cell to get a single measure of field stability. To attain a measurement of chance field overlap, the $s$ values were then calculated for each pairwise lap combination 
for each cell compared with all other cells. Finally, the field overlap of each cell was $z$-transformed using the chance overlap mean and SD to attain the degree of field overlap that was above chance $(Z>1.6)$.

\section{Results}

There were qualitative differences between how rats traversed the track forward and backward. For instance, rats tended to lower their nose toward the track and use whiskers to determine the edges (Movie 1). Moreover, the amount of time the rat spent in reverse ambulation (mean: $35.0 \mathrm{~min}, \mathrm{SEM} \pm 7.3$ ) tended to be longer compared with forward ambulation (mean: $18.8 \mathrm{~min}$, SEM \pm 3.9), although this difference was not statistically significant $\left(t_{(3)}=2.25, p=0.15\right)$. After extensive training, the rats did not show a statistical difference in their average velocities during forward (mean: $13.4 \mathrm{~cm} / \mathrm{s}$, SEM \pm 2.6) compared with backward (mean: $7.4 \mathrm{~cm} / \mathrm{s}$, SEM \pm 0.4$)$ runs $\left(t_{(3)}=\right.$ $3.46, p=0.08)$. Nonetheless, when comparing the highest $10 \%$ of velocities, there was a significant difference between running forward (mean: $41.6 \mathrm{~cm} / \mathrm{s}, \mathrm{SEM} \pm$ 6.5) and backward (mean: $15.8 \mathrm{~cm} / \mathrm{s}$, $\operatorname{SEM} \pm 1.1)$ conditions $\left(t_{(3)}=7.11, p<0.05\right)$. These data indicate that the rats spent more time running at faster speeds in the forward relative to the backward running conditions.

Only those cells with an information per spike score $>0.2$ (Skaggs et al., 1993), a spatial coherence score $>0.7$ (Muller et al., 1987), a minimum average of 10 spikes per lap, and a peak firing rate $>110 \%$ of the average firing rate for both running directions were included. A total of 437 of 1033 cells met the inclusion criteria. Figure 1 shows examples of single-unit activity. In traditional track-shuttling tasks, place-field activity is uncorrelated between inbound and outbound journeys (McNaughton et al., 1983), whereas place fields are omnidirectional in random foraging conditions (Muller et al., 1994). The reason for this discrepancy is unknown. The current study hypothesized that a rodent, in the same physical location, with the same head orientation, should have the same place-field activity independent of direction of travel. Therefore, place fields should be bidirectional as a rat ambulates forward and reverse on a linear track. To test this idea, a spatial population matrix was computed for every $0.33 \mathrm{~cm}$ spatial bin within each lap and then correlated with the matrices from all other laps and spatial bins (Gothard et al., 1996) within each running condition and between the forward and backward runs. Average bidirectional correlation values never exceeded 0.04 , and were significantly reduced relative to the correlation values across laps for the forward $\left(t_{(3)}=4.5, p<0.02\right)$ and backward $\left(t_{(3)}=12.9, p<0.001\right)$ running conditions alone. Contrary to the hypothesis, the data indicated place-field remapping between behavioral conditions, despite persistent head orientation (Fig. 2). In addition, hippocampal cells exhibited higher correlations over a larger area in reverse traversals relative to forward (Fig. 2A,B; $35.7 \mathrm{~cm}$ during backward runs, $17.9 \mathrm{~cm}$ for forward runs; $\left.t_{(3)}=5.8, p<0.01\right)$. The overall mean correlation across laps between the forward $(r=0.22)$ and backward $(r=$ 0.20 ) directions, however, was not significantly different from each other $\left(t_{(3)}=1.6, p=0.21\right)$, indicating similar stability for the population as a whole across laps in both conditions (Fig. 2C).

The population vectors of spatial activity for one lap can also be used to "reconstruct" the rat's position in other laps by finding the location of maximum correlation (Wilson and McNaughton, 1993). The position of maximum correlation tended to fall around unity in both forward and backward running conditions (Fig. 2D). Although place fields were larger during backward running, the mean reconstruction error between the forward and reverse directions was not significantly different $\left(t_{(3)}=0.8, p=\right.$ 0.47 ). This indicates that, despite reduced spatial specificity and decreased consistency in the locations of place-field centers across laps (see Materials and Methods), the population activity was able to update the rat's position similarly in both directions.

Although the population of hippocampal neurons on broad timescales is able to reconstruct the rodent's position in space, we were also interested to determine whether the short-time scale temporal dynamics were maintained during backward running. To explicitly measure the advancement of spikes relative to the hippocampal theta rhythm, we used three nonspatial analyses. By comparing the phase of spikes that lead an epoch of high firing rate versus spikes that fell on the tail end of a high firing rate epoch (Harris et al., 2002; see Materials and Methods) one can determine whether the phase of onset of spikes occurs later relative to the phase of offset bursts during forward and backward running (Fig. 3). In both the forward $\left(F_{(1,5125)}=66.76\right)$ and reverse $\left(F_{(1,4192)}=57.04\right)$ running conditions, the phase of spikes at the end of a high firing rate epoch occurred earlier than the spikes at the beginning of the high firing rate epoch $(p<000.1$; pairwise Mardia-Watson-Wheeler test).

Pyramidal cells showed theta-modulated firing in both forward (mean: 0.6 depth of modulation, SEM \pm 0.25 ) and backward (mean: 0.6 depth of modulation, SEM \pm 0.25 ) running conditions with no significant difference in the depth of modulation $\left(t_{(3)}=1.08, p=0.48\right)$. As initially noted by O'Keefe and 
A
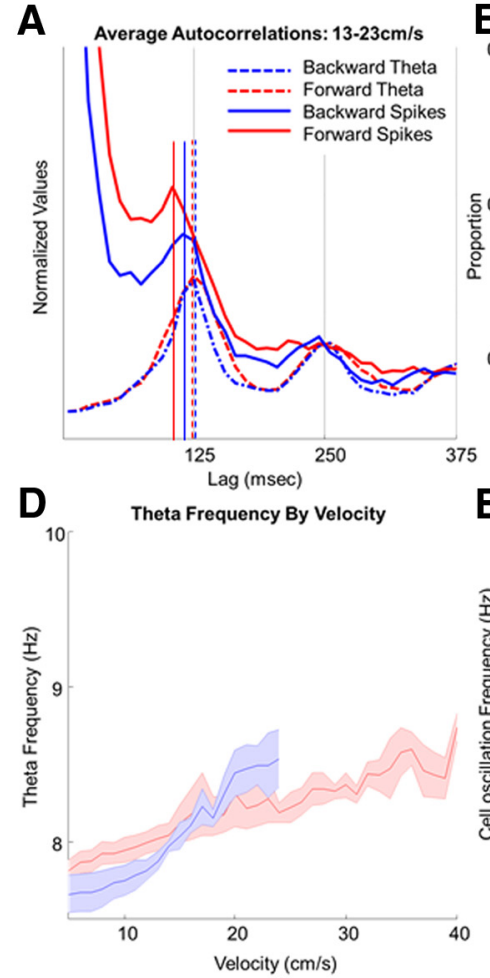

B

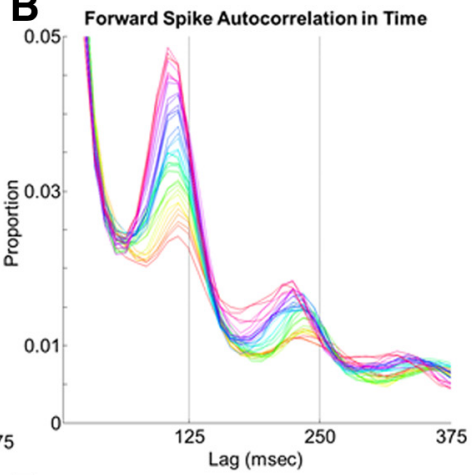

$\mathbf{E}$

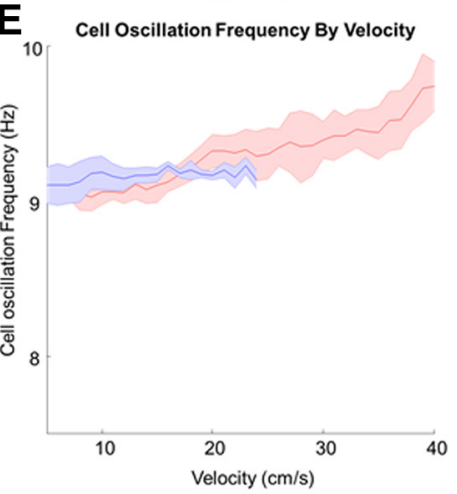

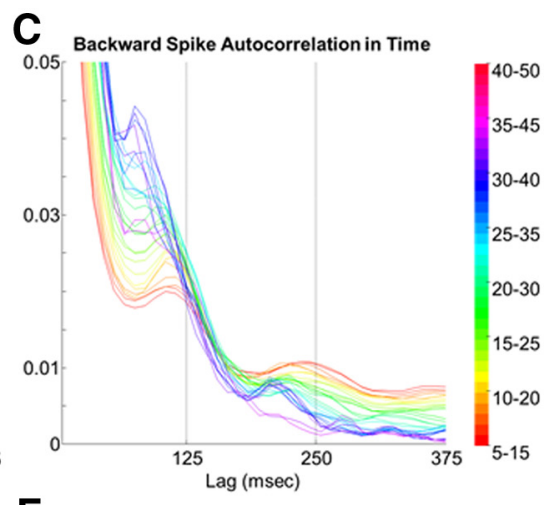

$\mathbf{F}$
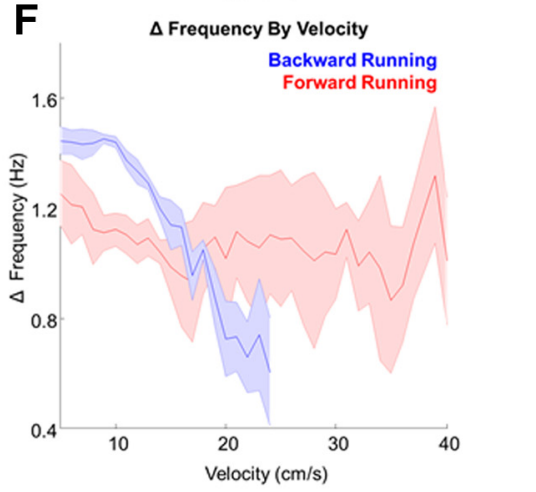

Figure 4. Spike and theta autocorrelogram frequency during forward and reverse running. $A$, The average autocorrelograms of the $13-23 \mathrm{~cm} / \mathrm{s}$ velocity bin for spikes (solid lines) and theta (dashed lines) during forward (red) and backward (blue) running. Only one velocity bin is shown to demonstrate the relationship between running speed and both of these parameters. The vertical lines indicate the lag from 0 of the first peak in the spike autocorrelogram (i.e., the interburst interval) and the first peak in the theta autocorrelogram (i.e., the duration of a single theta cycle). Note, that during forward and backward running, the first peak of the autocorrelogram occurs before the corresponding peak in theta. Thus, the bursting frequency is faster than theta frequency indicating that theta phase precession occurs in both movement conditions. $\boldsymbol{B}$, The mean spike autocorrelogram of principal cells during forward running. The different color traces correspond to the different velocity bins (cm/s) indicated on the scale bar on the right. $C$, Same as in $\boldsymbol{B}$ except for backward running. Note that fewer velocity bins are represented due to rats running slower in the backward condition relative to forward running. $\boldsymbol{D}$, The estimation of theta frequency by velocity calculated from the latency of the first peak in the theta autocorrelogram forward (red) and backward (blue) running conditions. The solid lines indicate the mean frequency across rats and the shading area represents $\pm 1 \mathrm{SEM}$. Slopes significantly increased with velocity (forward running: $0.02 \mathrm{cycles} / \mathrm{cm}$, $p<0.05$; backward running: 0.05 cycles/cm, $p<0.05$ ). $\boldsymbol{E}$, Same as in $\boldsymbol{D}$ except frequency ( $y$-axis) was estimated from the peaks of the cell autocorrelograms (that is, from the interburst interval) and is plotted as a function of running speed for forward (red) and backward (blue) running conditions. $\boldsymbol{F}$, The difference between cell oscillation and theta frequencies for different running conditions, across all velocities. Note that across all velocities the cell oscillation $(\boldsymbol{E})$ is significantly greater than the theta oscillation $(\boldsymbol{D})$, indicating theta phase precession.

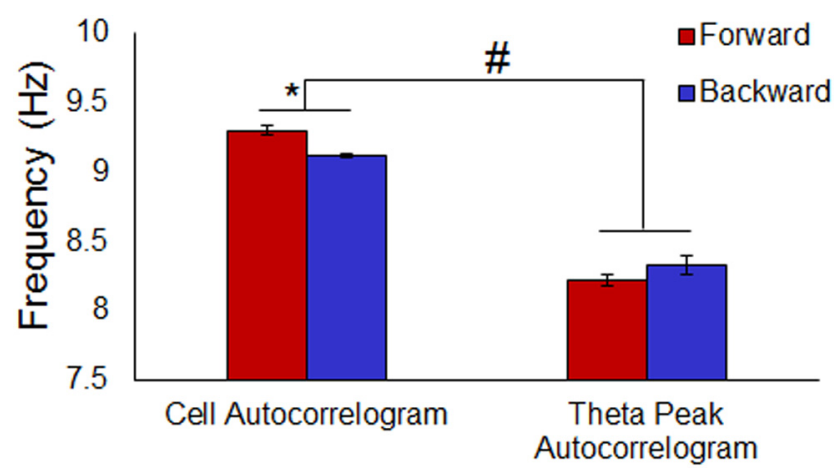

Figure 5. Mean autocorrelogram frequency of the cell spiking versus theta peaks. The $y$-axis shows the mean frequency of the cell and theta peaks autocorrelograms ( $x$-axis) for the forward (red) and backward (blue) running conditions. Running condition did not significantly affect the overall autocorrelogram frequency $(p<0.43)$, but the interaction between running condition and autocorrelogram was significant $(p<0.01)$. Post hoc analysis showed that during both forward and backward running there was a significant difference between the frequency of the spiking and theta autocorrelograms $(p<0.001$ for both comparisons; Tukey), indicative of theta phase precession. The frequency of the theta autocorrelogram did not significantly differ between running conditions $(p=0.33$; Tukey), but cell autocorrelogram frequency was significantly greater during forward running relative to backward $\left(p<0.05\right.$; Tukey); ${ }^{*} p<0.05$, \#p $<0.001$. Error bars indicate \pm 1 SEM.
Recce (1993), the occurrence of theta phase precession requires that the burst frequency of neurons is slightly faster than the hippocampal theta rhythm (Fig. 4). Therefore, it is possible to demonstrate that a neuron's spike time shifts relative to the theta oscillation if the frequency of the spike autocorrelogram is faster than the frequency of the autocorrelogram of theta peak times (Geisler et al., 2007). Figure 5 shows the mean values of the frequency of the autocorrelograms for the cells versus theta during forward and backward running. These frequency values were subject to a two-way factorial ANOVA with the factors of running condition (forward vs backward) and autocorrelogram (spikes vs theta). The main effect of autocorrelogram was significant $\left(F_{(1,70)}=541.56, p<0.001\right)$, such that the frequency of the spike autocorrelogram was significantly faster compared with theta. Running condition, however, did not significantly affect the overall autocorrelogram frequency $\left(F_{(1,70)}=0.57, p<0.43\right)$. The interaction between running condition and autocorrelogram was also significant $\left(F_{(1,70)}=12.47, p<0.01\right)$. Post hoc analysis showed that during both forward and backward running there was a significant difference between the frequency of the spike and theta autocorrelograms $(p<0.001$ for both comparisons; Tukey). This indicates that the cells precessed relative to theta in both running conditions. Importantly, the frequency of the theta autocorrelogram did not significantly differ between running conditions ( $p=0.33$; Tukey), but cell autocorrelogram fre- 
A

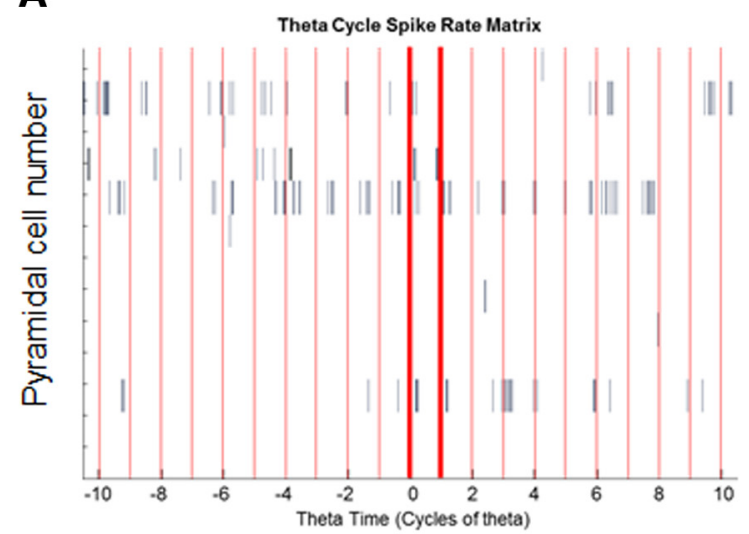

Correlation of the Reference Cycle to the Surrounding Cycles

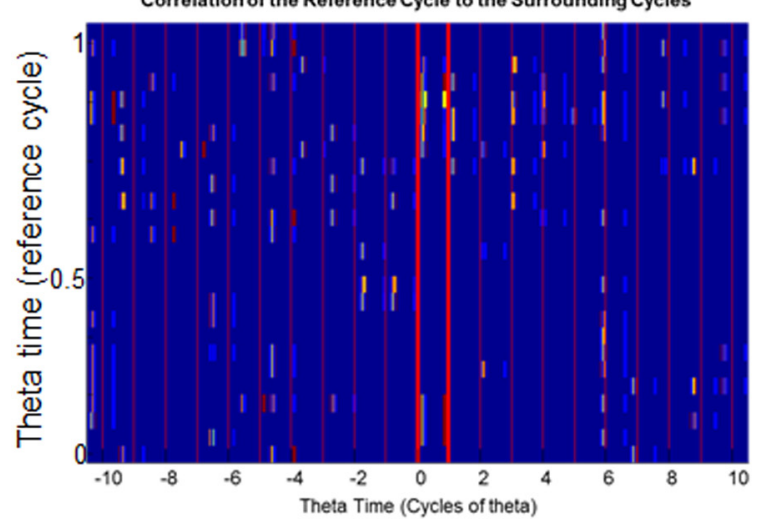

B

B

Rat Average Forward Theta time correlation

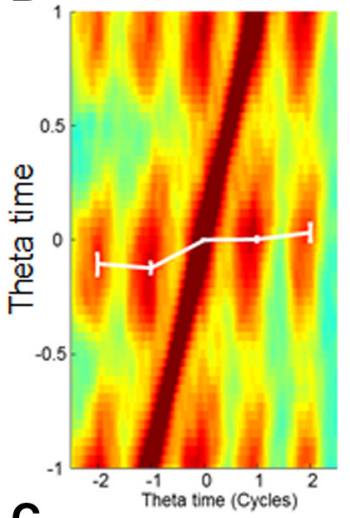

C

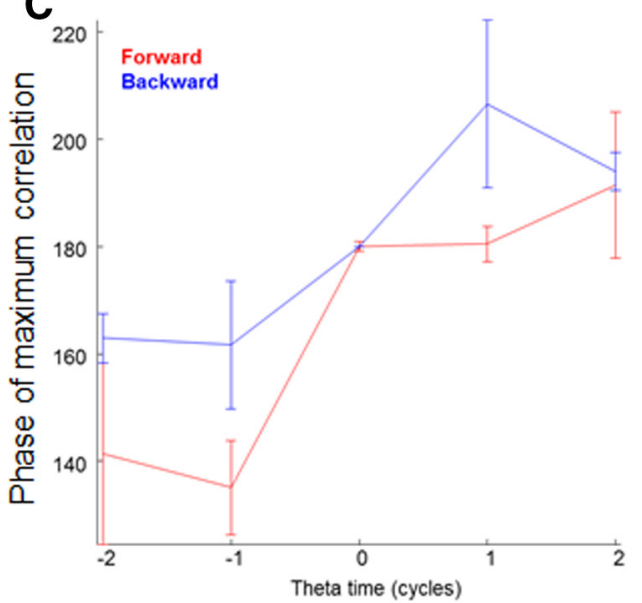

Figure 6. Theta phase precession of the CA1 neuronal population during forward and reverse running. A, Schematic of the method for creating population matrix theta-time autocorrelograms. For simplicity, only one cycle is used as a reference in this example (indicated by thick vertical red lines) although in actual analyses, every bin within a window of 21 theta cycles were correlated with every other bin. Gray vertical tick marks are the spike rasters for the different neurons. The number of spikes for each cell were binned by theta time for 20 cycles surrounding the reference cycle (boundaries marked by red lines). Every bin (columnar vector) of the central reference theta was correlated with every other vector to produce a theta-time autocorrelogram matrix (bottom). This matrix depicts the extent that cellular activity within a theta cycle correlates with other cycles. In the theta-time autocorrelogram matrix, the warmer colors correspond to theta phases within the reference cycle with higher correlation values across the different theta times represented over all 20 cycles of theta examined. Dark blue areas represent phase times in which no spikes occurred resulting in a nonexistent correlation or NaN value. Note that sparse vectors result in a sparse theta phase-time autocorrelogram matrix, and that the cell dimension in the spike-rate matrix drops out, leaving a theta time autocorrelogram with the units of phase. $\boldsymbol{B}$, The average of all theta-time autocorrelation matrices across rats. By averaging over multiple theta cycles, the bins with NaN values dropout and the overall correlation averages develop high resolution. The population vector theta-time autocorrelograms are shown for the forward (left) and backward (right) running conditions. $C$, The $x$-axis shows theta time over four cycles and the $y$-axis is the phase of maximum correlation. The phase of maximum correlation significantly changed across adjacent theta cycles. This indicates that the CA1 neuronal population exhibits theta phase precession, in both forward and backward running conditions.

quency was significantly greater during forward running relative to backward ( $p<0.05$; Tukey). This is consistent with the observation that place-field size was significantly larger during backward running relative to forward as indicated by the spatial population vector correlation.

To evaluate the effect of backward ambulation on the theta phase precession across the recorded ensemble, population activity was temporally binned relative to the hippocampal theta oscillation and correlated across multiple theta cycles (Fig. 6A). The phase of maximum correlation for the trough of the two cycles preceding and following the reference cycle was then calculated. This method quantifies how the population activity changes over successive theta cycles. During forward running laps, the average phase shift over five cycles was $50^{\circ}$, which was significantly different from $0\left(t_{(3)}=3.2, p<0.05\right.$; one-sample $t$ test $)$. Moreover, during the backward running laps the total phase shift was $31^{\circ}$ and this was also significantly different from $0\left(t_{(3)}=5.8, p<\right.$ 0.01 ; one-sample $t$ test). The forward and backward phase shifts were not statistically different from each other, however $\left(t_{(3)}=\right.$ $1.38, p<0.3$ ), suggesting that the population dynamics of CA1 neurons show theta phase precession in both running directions (Fig. 6B, C).

\section{Discussion}

By decoupling a rodent's movement direction from its head orientation, the current experiment tested two competing attractor network models. One class of models uses head-direction, and self-motion signals, to shift the ensemble of active neurons to fields located ahead of the rodent during movement (Redish and Touretzky, 1997; Samsonovich and McNaughton, 1997; McNaughton et al., 2006). The second model proposes that a velocity vector, potentially from the parietal cortex, provides directional input to the hippocampal network (Conklin and Eliasmith, 2005). If a rat were to run backward, in opposition to the direction it is facing, there are two potential outcomes, each lending support to one model over the other. The head-direction hypothesis would predict that hippocampal spatial activity should be distorted or destroyed during backward running. Alternatively, the posterior parietal model of hippocampal updating suggests that the place code should be maintained regardless 
of direction of ambulation (Conklin and Eliasmith, 2005). This is not to suggest that other brain regions involved in movement information, such as the prefrontal cortex, striatum, or cerebellum, are not also critical for updating hippocampal activity patterns.

Although there was a higher variance of center-of-mass across laps during backward running, the rat's position reconstruction could be obtained from the population of active cells. Moreover, this was comparable to the reconstruction accuracy obtained during forward running. Furthermore, intact theta phase precession observed during both forward and backward running, as indicated by two independent single-cell analyses and a population-based temporal vector correlation measure, is evidence that the network activity can be appropriately updated on short-time scales as well. Therefore, the present data favor the parietal mechanism of updating.

As place fields were significantly larger in backward compared with forward running, which could be interpreted as a distortion of the map, this finding could potentially support models of head-direction mechanisms for hippocampal updating. An alternate explanation, however, is that the increase in place-field size is a result of contradictory velocity processing between the parietal cortex (Whitlock et al., 2012) and the head-direction cells, which are known to increase their firing rates with linear velocity (Taube et al., 1990). In the parietal model of hippocampal updating, head-direction neurons govern the velocity input into the network (Conklin and Eliasmith, 2005). As sensitivity to incoming self-motion information, inferred by velocity-firing rate relationships, has been suggested to govern hippocampal place-field size (Maurer et al., 2005; Terrazas et al., 2005), it is feasible that the head-direction system manages the flow of parietal velocity input to the hippocampal network. During reverse ambulation, this signal is mitigated. Moreover, place cells have been described as speed-controlled oscillators, in which their autocorrelation burst frequency increases with velocity (O'Keefe and Recce, 1993; Geisler et al., 2007). In line with this idea, pyramidal neurons were found to oscillate at a slower frequency during backward running relative to forward running, supporting the idea that there was attenuated velocity information during backward movement.

In a recent experiment by Cei et al. (2014), rodents were trained to run forward on a treadmill. The treadmill could be moved forward in space, congruent with the rat's head-direction and running-direction, or it could move backward, incongruent with the head direction and running direction of the rat. In this experiment, the posterior parietal velocity information is not in conflict with head-direction, as the rat is always ambulating forward even when the train is moving backward. One interesting difference between the current data and those in Cei et al. (2014) is that in the present study CA1 place cell activity was unidirectional as opposed to bidirectional. As there is a population of neurons in the posterior parietal cortex that show activity correlated with "simple motion states" (McNaughton et al., 1994; Whitlock et al., 2012), one explanation for the disparate findings regarding direction-selectivity could be differences in posterior parietal neuron activity between the treadmill and reverse ambulation conditions. As posterior parietal cortical neurons have altered activity in north- and south-bound routes in a hairpin maze (Whitlock et al., 2012), their directional selectivity may be responsible for unidirectional place fields on linear tracks. When the movement vector is maintained, such as during treadmill running, parietal information is hypothetically maintained, resulting in bidirectional place fields (Cei et al., 2014). Given that the posterior parietal activity precedes that of the onset of movement by up to $500 \mathrm{~ms}$ (Gold and Shadlen, 2000; Whitlock et al., 2012), it is also possible that a signal from this region is responsible for driving the observed predictive hippocampal sweeps (Johnson and Redish, 2007), as well as forward and reverse reactivation observed at the ends of a track (Foster and Wilson, 2006; Diba and Buzsàki, 2007). Finally, as the route-specific correlations of posterior parietal neurons are scalable in size (Nitz, 2006) and capable of holding multiple reference frames at a single moment (Nitz, 2012), it is possible that this cortical input contributes to the scaling of hippocampal place fields and grid cells in conditions when a familiar environment is compressed or stretched (O'Keefe and Burgess, 1996; Gothard et al., 2001; Huxter et al., 2003; Barry et al., 2007).

Numerous empirical data and theoretical models indicate that the sequential activation of place cells within a theta cycle is responsible for the phenomenon of theta phase precession (Dragoi and Buzsàki, 2006; Foster and Wilson, 2007; Maurer and McNaughton, 2007; Maurer et al., 2012) and perhaps theta itself (Geisler et al., 2010). Thus, considering the current data in terms of mechanisms for governing temporal sequence dynamics may be more pertinent than determining which theta phase precession model best accounts for the these results. Fortunately, models of hippocampal theta phase precession complement each other when it comes to understanding the phenomenon of ensemble sequence encoding. The intrinsic oscillation of neurons (O'Keefe and Recce, 1993; Kamondi et al., 1998; Bose et al., 2000; Harris et al., 2002; Lengyel et al., 2003) can be synchronized via strong synaptic network connections (Jensen and Lisman, 1996; Tsodyks et al., 1996) and precisely controlled by reciprocal inhibitory-excitatory connections (Wallenstein and Hasselmo, 1997; Mehta et al., 2002). Thus, each component of the prior models can be integrated to support the self-organized dynamic patterns (Kelso, 1995) critical for ensemble sequence encoding.

There is recent experimental evidence to validate the idea that there is dramatic interconnectivity between the models. For example, driving parvalbumin interneurons in a network with an optical chirp stimulus can rhythmically entrain pyramidal neurons; an effect dependent on hyperpolarization-activated cyclic nucleotide-gated (HCN) channel function (Stark et al., 2013). Moreover, the recent observation that neurons maintain their firing rate magnitude across environments and behavioral states supports the idea of a preconfigured network capable of asymmetric neuronal activation (i.e., "trajectories"; Pastalkova et al., 2008; Mizuseki and Buzsàki, 2014) In light of these findings, inhibition and excitation models of phase precession, along with intrinsic oscillation dynamics and asymmetric network connectivity models, can be integrated to propagate a sequence of activity across the network Under this framework, at the beginning of each theta cycle, input into the hippocampus activates a specific cell assembly with the magnitude of activation proportional to the velocity input (Conklin and Eliasmith, 2005; Maurer et al., 2012). This "node of attraction" (Izhikevich, 2007) is transient, limited by mechanisms, such as the distribution of pyramidal cell HCN channels (Giocomo and Hasselmo, 2008; Dougherty et al., 2013), and feedback projections from inhibitory interneurons (Marshall et al., 2002; Maurer et al., 2006a; Geisler et al., 2007). Provided a vector of displacement (i.e., heading and velocity as described by Conklin and Eliasmith, 2005), and the asymmetric firing rate biases of the network (Mizuseki and Buzsàki, 2014), the network activity moves toward he next most stable state. In this view, a chain reaction occurs in which the total number of distinct assemblies activated within a single theta cycle is propor- 
tional to the magnitude of the velocity input (Conklin and Eliasmith, 2005; Maurer et al., 2012). Notably, as the signal propagates within the theta cycle, each sequential state is less stable than its predecessor due to a loss of excitation (partially due to feedback inhibition). Moreover, the amount of time that each assembly is active becomes progressively shorter, shifting later in the theta cycle. The propagation of activity therefore effectively accelerates across assemblies while simultaneously losing strength until it collapses. This description effectively accounts for the heteroskedasticity in phase-position portraits (cells are active for significantly longer periods of time early in the theta cycle), and bimodality (the activity of the network accelerates away from the initial state). This also accounts for the current finding that the short-time scale dynamics of the hippocampus are maintained during backward running. Together, the present data show that CA1 ensemble sequence encoding persists even when the sequence is in the direction opposite of the animal's orientation. This may be due to self-organized hippocampal dynamics in conjunction with parietal cortical input.

\section{References}

Barry C, Hayman R, Burgess N, Jeffery KJ (2007) Experience-dependent rescaling of entorhinal grids. Nat Neurosci 10:682-684. CrossRef Medline

Battaglia FP, Sutherland GR, McNaughton BL (2004) Local sensory cues and place cell directionality: additional evidence of prospective coding in the hippocampus. J Neurosci 24:4541-4550. CrossRef Medline

Belluscio MA, Mizuseki K, Schmidt R, Kempter R, Buzsáki G (2012) Crossfrequency phase-phase coupling between theta and gamma oscillations in the hippocampus. J Neurosci 32:423-435. CrossRef Medline

Bose A, Booth V, Recce M (2000) A temporal mechanism for generating the phase precession of hippocampal place cells. J Comput Neurosci 9:5-30. CrossRef Medline

Burgess N, Recce M, O’Keefe J (1994) A model of hippocampal function. Neural Netw 7:1065-1081. CrossRef

Burke SN, Maurer AP, Yang Z, Navratilova Z, Barnes CA (2008) Glutamate receptor-mediated restoration of experience-dependent place field expansion plasticity in aged rats. Behav Neurosci 122:535-548. CrossRef Medline

Burke SN, Maurer AP, Nematollahi S, Uprety AR, Wallace JL, Barnes CA (2011) The influence of objects on place field expression and size in distal hippocampal CA1. Hippocampus 21:783-801. CrossRef Medline

Burke SN, Chawla MK, Penner MR, Crowell BE, Worley PF, Barnes CA, McNaughton BL (2005) Differential encoding of behavior and spatial context in deep and superficial layers of the neocortex. Neuron 45:667674. CrossRef Medline

Buzsàki G, Eidelberg E (1983) Phase relations of hippocampal projection cells and interneurons to theta activity in the anesthetized rat. Brain Res 266:334-339. CrossRef Medline

Buzsàki G, Leung LW, Vanderwolf CH (1983) Cellular bases of hippocampal EEG in the behaving rat. Brain Res 287:139-171. Medline

Cei A, Girardeau G, Drieu C, Kanbi KE, Zugaro M (2014) Reversed theta sequences of hippocampal cell assemblies during backward travel. Nat Neurosci 17:719-724. CrossRef Medline

Conklin J, Eliasmith C (2005) A controlled attractor network model of path integration in the rat. J Comput Neurosci 18:183-203. CrossRef Medline

Csicsvari J, Hirase H, Czurko A, Buzsáki G (1998) Reliability and state dependence of pyramidal cell-interneuron synapses in the hippocampus: an ensemble approach in the behaving rat. Neuron 21:179-189. CrossRef Medline

Diba K, Buzsáki G (2007) Forward and reverse hippocampal place-cell sequences during ripples. Nat Neurosci 10:1241-1242. CrossRef Medline

Diba K, Buzsáki G (2008) Hippocampal network dynamics constrain the time lag between pyramidal cells across modified environments. J Neurosci 28:13448-13456. CrossRef Medline

Dougherty KA, Nicholson DA, Diaz L, Buss EW, Neuman KM, Chetkovich DM, Johnston D (2013) Differential expression of HCN subunits alters voltage-dependent gating of $h$-channels in CAl pyramidal neurons from dorsal and ventral hippocampus. J Neurophysiol 109:1940-1953. CrossRef Medline
Dragoi G, Buzsáki G (2006) Temporal encoding of place sequences by hippocampal cell assemblies. Neuron 50:145-157. CrossRef Medline

Ekstrom AD, Meltzer J, McNaughton BL, Barnes CA (2001) NMDA receptor antagonism blocks experience-dependent expansion of hippocampal "place fields." Neuron 31:631-638. CrossRef Medline

Foster DJ, Wilson MA (2006) Reverse replay of behavioural sequences in hippocampal place cells during the awake state. Nature 440:680-683. CrossRef Medline

Foster DJ, Wilson MA (2007) Hippocampal theta sequences. Hippocampus 17:1093-1099. CrossRef Medline

Geisler C, Robbe D, Zugaro M, Sirota A, Buzsáki G (2007) Hippocampal place cell assemblies are speed-controlled oscillators. Proc Natl Acad Sci U S A 104:8149-8154. CrossRef Medline

Geisler C, Diba K, Pastalkova E, Mizuseki K, Royer S, Buzsáki G (2010) Temporal delays among place cells determine the frequency of population theta oscillations in the hippocampus. Proc Natl Acad Sci U S A 107: 7957-7962. CrossRef Medline

Giocomo LM, Hasselmo ME (2008) Time constants of h current in layer ii stellate cells differ along the dorsal to ventral axis of medial entorhinal cortex. J Neurosci 28:9414-9425. CrossRef Medline

Gold JI, Shadlen MN (2000) Representation of a perceptual decision in developing oculomotor commands. Nature 404:390-394. CrossRef Medline

Gothard KM, Skaggs WE, McNaughton BL (1996) Dynamics of mismatch correction in the hippocampal ensemble code for space: interaction between path integration and environmental cues. J Neurosci 16:80278040. Medline

Gothard KM, Hoffman KL, Battaglia FP, McNaughton BL (2001) Dentate gyrus and cal ensemble activity during spatial reference frame shifts in the presence and absence of visual input. J Neurosci 21:7284-7292. Medline

Hafting T, Fyhn M, Molden S, Moser MB, Moser EI (2005) Microstructure of a spatial map in the entorhinal cortex. Nature 436:801-806. CrossRef Medline

Harris KD (2005) Neural signatures of cell assembly organization. Nat Rev Neurosci 6:399-407. CrossRef Medline

Harris KD, Henze DA, Hirase H, Leinekugel X, Dragoi G, Czurkó A, Buzáaki G (2002) Spike train dynamics predicts theta-related phase precession in hippocampal pyramidal cells. Nature 417:738-741. CrossRef Medline

Huxter JR, Senior TJ, Allen K, Csicsvari J (2008) Theta phase-specific codes for two-dimensional position, trajectory and heading in the hippocampus. Nat Neurosci 11:587-594. CrossRef Medline

Huxter J, Burgess N, O'Keefe J (2003) Independent rate and temporal coding in hippocampal pyramidal cells. Nature 425:828-832. CrossRef Medline

Izhikevich EM (2007) Dynamical systems in neuroscience. Cambridge, Massachusetts: MIT.

Jensen O, Lisman JE (1996) Hippocampal CA3 region predicts memory sequences: accounting for the phase precession of place cells. Learn Mem 3:279-287. CrossRef Medline

Johnson A, Redish AD (2007) Neural ensembles in CA3 transiently encode paths forward of the animal at a decision point. J Neurosci 27:1217612189. CrossRef Medline

Kamondi A, Acsády L, Wang XJ, Buzsáki G (1998) Theta oscillations in somata and dendrites of hippocampal pyramidal cells in vivo: activitydependent phase-precession of action potentials. Hippocampus 8:244261. CrossRef Medline

Kelso, JS (1995) Dynamic patterns: the self-organization of brain and behavior. Boston: MIT.

Kempter R, Leibold C, Buzsáki G, Diba K, Schmidt R (2012) Quantifying circular-linear associations: hippocampal phase precession. J Neurosci Methods 207:113-124. CrossRef Medline

Knierim JJ, Kudrimoti HS, McNaughton BL (1995) Place cells, head direction cells, and the learning of landmark stability. J Neurosci 15:16481659. Medline

Lee I, Rao G, Knierim JJ (2004) A double dissociation between hippocampal subfields: differential time course of CA3 and CA1 place cells for processing changed environments. Neuron 42:803-815. CrossRef Medline

Lengyel M, Szatmáry Z, Erdi P (2003) Dynamically detuned oscillations account for the coupled rate and temporal code of place cell firing. Hippocampus 13:700-714. CrossRef Medline

Lisman J, Buzsáki G (2008) A neural coding scheme formed by the com- 
bined function of gamma and theta oscillations. Schizophr Bull 34:974980. CrossRef Medline

Lisman JE, Idiart MA (1995) Storage of $7+/-2$ short-term memories in oscillatory subcycles. Science 267:1512-1515. CrossRef Medline

Markus EJ, Barnes CA, McNaughton BL, Gladden VL, Skaggs WE (1994) Spatial information content and reliability of hippocampal CA1 neurons: effects of visual input. Hippocampus 4:410-421. CrossRef Medline

Marshall L, Henze DA, Hirase H, Leinekugel X, Dragoi G, Buzsáki G (2002) Hippocampal pyramidal cell-interneuron spike transmission is frequency dependent and responsible for place modulation of interneuron discharge. J Neurosci 22:RC197. Medline

Maurer AP, McNaughton BL (2007) Network and intrinsic cellular mechanisms underlying theta phase precession of hippocampal neurons. Trends Neurosci 30:325-333. CrossRef Medline

Maurer AP, Vanrhoads SR, Sutherland GR, Lipa P, McNaughton BL (2005) Self-motion and the origin of differential spatial scaling along the septotemporal axis of the hippocampus. Hippocampus 15:841-852. CrossRef Medline

Maurer AP, Cowen SL, Burke SN, Barnes CA, McNaughton BL (2006a) Phase precession in hippocampal interneurons showing strong functional coupling to individual pyramidal cells. J Neurosci 26:13485-13492. CrossRef Medline

Maurer AP, Cowen SL, Burke SN, Barnes CA, McNaughton BL (2006b) Organization of hippocampal cell assemblies based on theta phase precession. Hippocampus 16:785-794. CrossRef Medline

Maurer AP, Burke SN, Lipa P, Skaggs WE, Barnes CA (2012) Greater running speeds result in altered hippocampal phase sequence dynamics. Hippocampus 22:737-747. CrossRef Medline

McNaughton BL, Barnes CA, O'Keefe J (1983) The contributions of position, direction, and velocity to single unit activity in the hippocampus of freely-moving rats. Exp Brain Res 52:41-49. Medline

McNaughton BL, Mizumori SJ, Barnes CA, Leonard BJ, Marquis M, Green EJ (1994) Cortical representation of motion during unrestrained spatial navigation in the rat. Cereb Cortex 4:27-39. CrossRef Medline

McNaughton BL, Battaglia FP, Jensen O, Moser EI, Moser MB (2006) Path integration and the neural basis of the "cognitive map." Nat Rev Neurosci 7:663-678. CrossRef Medline

Mehta MR, Barnes CA, McNaughton BL (1997) Experience-dependent, asymmetric expansion of hippocampal place fields. Proc Natl Acad Sci U S A 94:8918-8921. CrossRef Medline

Mehta MR, Quirk MC, Wilson MA (2000) Experience-dependent asymmetric shape of hippocampal receptive fields. Neuron 25:707-715. CrossRef Medline

Mehta MR, Lee AK, Wilson MA (2002) Role of experience and oscillations in transforming a rate code into a temporal code. Nature 417:741-746. CrossRef Medline

Mizuseki K, Buzsaki G (2014) Theta oscillations decrease spike synchrony in the hippocampus and entorhinal cortex. Philos Trans R Soc Lond B Biol Sci 369:20120530. CrossRef Medline

Muller RU, Kubie JL, Ranck JB Jr (1987) Spatial firing patterns of hippocampal complex-spike cells in a fixed environment. J Neurosci 7:19351950. Medline

Muller RU, Bostock E, Taube JS, Kubie JL (1994) On the directional firing properties of hippocampal place cells. J Neurosci 14:7235-7251. Medline

Nitz DA (2006) Tracking route progression in the posterior parietal cortex. Neuron 49:747-756. CrossRef Medline

Nitz DA (2012) Spaces within spaces: rat parietal cortex neurons register position across three reference frames. Nat Neurosci 15:1365-1367. CrossRef Medline
O'Keefe J, Burgess N (1996) Geometric determinants of the place fields of hippocampal neurons. Nature 381:425-428. CrossRef Medline

O'Keefe J, Dostrovsky J (1971) The hippocampus as a spatial map: preliminary evidence from unit activity in the freely-moving rat. Brain Res 34: 171-175. CrossRef Medline

O'Keefe J, Recce ML (1993) Phase relationship between hippocampal place units and the EEG theta rhythm. Hippocampus 3:317-330. CrossRef Medline

Pastalkova E, Itskov V, Amarasingham A, Buzsáki G (2008) Internally generated cell assembly sequences in the rat hippocampus. Science 321:13221327. CrossRef Medline

Ranck JB Jr (1973) Studies on single neurons in dorsal hippocampal formation and septum in unrestrained rats: I. Behavioral correlates and firing repertoires. Exp Neurol 41:461-531. Medline

Redish AD, Touretzky DS (1997) Cognitive maps beyond the hippocampus. Hippocampus 7:15-35. CrossRef Medline

Samsonovich A, McNaughton BL (1997) Path integration and cognitive mapping in a continuous attractor neural network model. J Neurosci 17:5900-5920. Medline

Shen J, Barnes CA, McNaughton BL, Skaggs WE, Weaver KL (1997) The effect of aging on experience-dependent plasticity of hippocampal place cells. J Neurosci 17:6769-6782. Medline

Skaggs WE, McNaughton BL, Wilson MA, Barnes CA (1996) Theta phase precession in hippocampal neuronal populations and the compression of temporal sequences. Hippocampus 6:149-172. CrossRef Medline

Skaggs WE, McNaughton BL, Gothard KG, Markus EJ (1993) An information theoretic approach to deciphering the hippocampal code. In: Progress in neural information processing systems (Hansen SJ, Cowan JD, Giles CL, eds), pp 1030-1037. San Mateo, CA: Kauffman.

Stark E, Eichler R, Roux L, Fujisawa S, Rotstein HG, Buzsáki G (2013) Inhibition-induced theta resonance in cortical circuits. Neuron 80:12631276. CrossRef Medline

Taube JS, Muller RU, Ranck JB Jr (1990) Head-direction cells recorded from the postsubiculum in freely moving rats: II. Effects of environmental manipulations. J Neurosci 10:436-447. Medline

Terrazas A, Krause M, Lipa P, Gothard KM, Barnes CA, McNaughton BL (2005) Self-motion and the hippocampal spatial metric. J Neurosci 25: 8085-8096. CrossRef Medline

Thompson LT, Best PJ (1990) Long-term stability of the place-field activity of single units recorded from the dorsal hippocampus of freely behaving rats. Brain Res 509:299-308. CrossRef Medline

Tsodyks MV, Skaggs WE, Sejnowski TJ, McNaughton BL (1996) Population dynamics and theta rhythm phase precession of hippocampal place cell firing: a spiking neuron model. Hippocampus 6:271-280. CrossRef Medline

Vanderwolf $\mathrm{CH}$ (1969) Hippocampal electrical activity and voluntary movement in the rat. Electroencephalogr Clin Neurophysiol 26:407-418. CrossRef Medline

Wallenstein GV, Hasselmo ME (1997) GABAergic modulation of hippocampal population activity: sequence learning, place field development, and the phase precession effect. J Neurophysiol 78:393-408. Medline

Whitlock JR, Pfuhl G, Dagslott N, Moser MB, Moser EI (2012) Functional split between parietal and entorhinal cortices in the rat. Neuron 73:789802. CrossRef Medline

Wilson MA, McNaughton BL (1993) Dynamics of the hippocampal ensemble code for space. Science 261:1055-1058. CrossRef Medline

Yamaguchi Y, Aota Y, McNaughton BL, Lipa P (2002) Bimodality of theta phase precession in hippocampal place cells in freely running rats. J Neurophysiol 87:2629-2642. Medline 